\title{
Pc2 EMIC waves generated high off the equator in the dayside outer magnetosphere
}

\author{
Y. H. Liu, ${ }^{1,2}$ B. J. Fraser, ${ }^{1}$ and F. W. Menk ${ }^{1}$ \\ Received 12 July 2012; revised 25 July 2012; accepted 25 July 2012; published 7 September 2012.
}

[1] It is generally accepted that electromagnetic ion cyclotron (EMIC) waves are generated around the equatorial regions and propagate toward the high latitude ionospheres in both hemispheres. Here we describe a prolonged EMIC wave event in the Pc2 (0.1-0.2 Hz) frequency band above the $\mathrm{He}^{+}$cyclotron frequency detected by the four Cluster satellites as they traversed sunward from $L \sim 13$ in the outer magnetosphere to the magnetopause, over $13^{\circ}-20^{\circ}$ magnetic latitude north of the equator and across the high latitude cusp region near local magnetic noon. Wave packet energy propagated dominantly along the geomagnetic field direction, confirming this was a traveling EMIC wave rather than a toroidal field line resonance. The energy packets propagated in alternating directions rather than uni-directionally from the equator, implying the wave source was located in a high latitude region away from the equator, where a minimum in the $\mathrm{B}$ field is located. The CIS-CODIF $\mathrm{H}^{+}$ion data provided evidence that the waves were generated locally via the ion cyclotron instability. We believe the off-equatorial minimum magnetic field regions may be important source regions for these waves in the outer magnetosphere. Citation: Liu, Y. H., B. J. Fraser, and F. W. Menk (2012), Pc2 EMIC waves generated high off the equator in the dayside outer magnetosphere, Geophys. Res. Lett., 39, L17102, doi:10.1029/2012GL053082.

\section{Introduction}

[2] Pc1 $(0.2-5 \mathrm{~Hz})$ and Pc2 $(0.1-0.2 \mathrm{~Hz})$ electromagnetic ion cyclotron (EMIC) waves are often observed on closed magnetic field lines in Earth's magnetosphere, with wave sources generally believed to be located near the equatorial plane [Anderson et al., 1992a, 1992b; Fraser and Nguyen, 2001]. For a given dipole field line the magnetic field strength is a minimum near the equator. There will be minima in both the parallel energetic trapped $\mathrm{keV}$ particle energy and the wave group velocity around the equator, favorable for triggering the ion cyclotron instability and convective wave growth [Cornwall, 1965; Mauk, 1982]. The waves then appear to propagate in both directions along field lines within $\pm 11^{\circ}$ of the equator, and in a single direction

\footnotetext{
${ }^{1}$ Center for Space Physics, University of Newcastle, Callaghan, New South Wales, Australia.

${ }^{2}$ Polar Atmosphere and Space Physics Department, Polar Research Institute of China, Shanghai, China.

Corresponding author: Y. Liu, Polar Atmosphere and Space Physics Department, Polar Research Institute of China, 451 Jinqiao Rd., Shanghai 200129, China. (liuyonghua@hotmail.com)

C2012. American Geophysical Union. All Rights Reserved. 0094-8276/12/2012GL053082
}

toward the high latitude ionospheres beyond this latitude [Loto'aniu et al., 2005].

[3] In the near-noon outer magnetosphere geomagnetic field lines are compressed and distorted from dipole geometry due to solar wind dynamic pressure. The minimum in geomagnetic field strength does not then occur around the equator but at higher off-equatorial distances in both hemispheres, forming localized minimum B field environments [Shabansky, 1971]. Such a minimum B field can also confine charged particles undergoing mirror reflection and so may be a favorable region for the excitation of waves via wave-particle interaction, similar to the process by which waves are generated around the equator [Shabansky, 1971; McCollough et al., 2010; Antonova et al., 2011]. Tsurutani and Smith [1977] showed that electron cyclotron VLF waves are generated locally at high latitude minimum B field locations with frequency partly overlapping the range observed at the equator.

[4] Encouraged by these results for electron cyclotron waves and the simulations by McCollough et al. [2010] we may expect that EMIC waves can also be excited at high latitude, i.e., high off the equator, in minimum B field regions. In this paper we report observations of a prolonged Pc2 wave event by the four Cluster satellites in the high latitude magnetosphere near magnetic noon. To our knowledge, this is the first direct evidence of EMIC wave excitation in the minimum $B$ field region off the equator and suggests an important source region for $\mathrm{Pc} 2$ waves in the outer magnetosphere.

\section{Instruments}

[5] In this paper we focus on a long duration Pc2 wave event recorded near local noon by the four Cluster spacecraft as they traveled outward through the high latitude dayside magnetosphere. These spacecraft were launched in July 2000 into a polar orbit, with an orbital period of 57 hours and perigee and apogee of 4.0 and 19.6 Earth radii $\left(R_{E}\right)$, respectively [Escoubet et al., 2001]. The waves reported here were measured by the fluxgate magnetometer (FGM) sampled at $64 \mathrm{~Hz}$ [Balogh et al., 1997] in the geocentric solar ecliptic (GSE) coordinate system, resampled at $1 \mathrm{~Hz}$ then transformed into a field-aligned coordinate system (FAS) for analysis. The FAS system has the z-axis directed along the magnetic field equilibrium direction, which is derived by averaging over 600 data points covering a 10-minute time interval. The $y$-axis is determined by $\mathbf{z} \times \mathbf{r}$, perpendicular to the $\mathrm{z}$ direction and toward the east, and the $\mathrm{x}$-axis is given by $\mathbf{y} \times \mathbf{z}$, pointing out of the magnetosphere. Here $\mathbf{r}$ is the unit vector pointing out from the center of the Earth.

[6] We also present electric field data from the Electric Field and Waves (EFW) instrument [Gustafsson et al., 2001], 


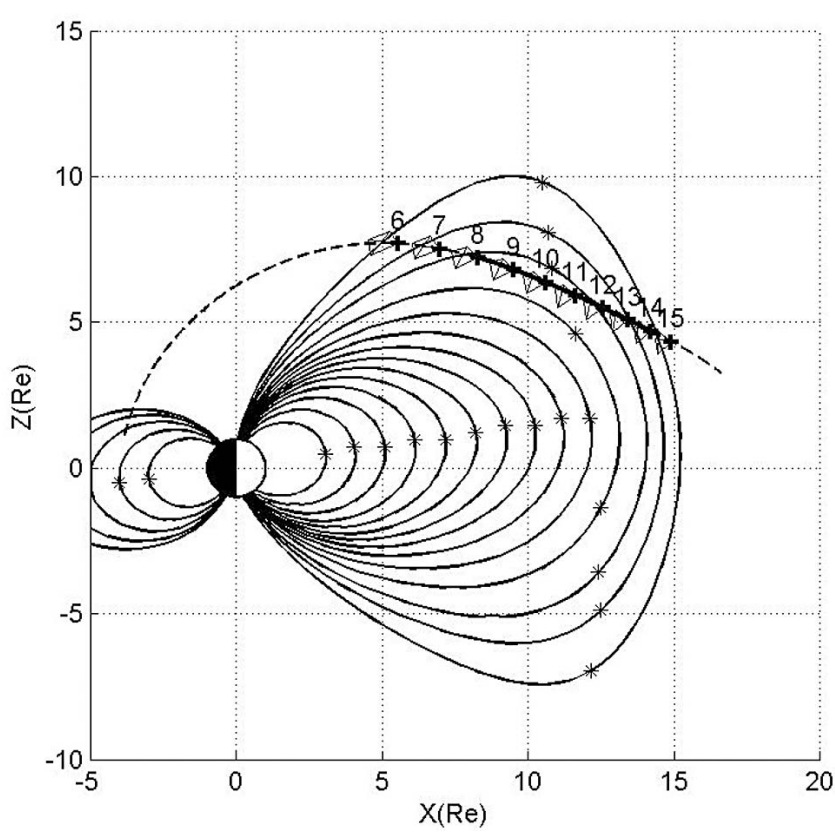

Figure 1. Cluster orbit track and magnetospheric configuration in the X-Z plane on 16 February 2004 based on calculations from the Tsyganenko [1996] model. Coordinates are shown in the Geocentric Solar Magnetic (GSM) system and in Earth radii. The Pc2 waves were observed in the time interval $0800-1430$ UT marked by the thick solid line. Universal time in hours is indicated by numbers along the orbit track, and the location of satellite $\mathrm{C} 3$ is denoted by black ' + ' symbols. The tetrahedron symbols represent the formation of the four Cluster satellites, while the distances between the satellites have been enlarged by 10 times. The points with local minimum B field along a field line are marked by black asterisk symbols.

which were measured at $25 \mathrm{~Hz}$ and resampled at $1 \mathrm{~Hz}$ in order to estimate the Poynting flux vector for the Pc2 waves. Finally, we make use of particle data from the CODIF instrument, which is a high-sensitivity mass-resolving spectrometer with $360^{\circ}$ field-of-view, to measure the full threedimensional distribution function of the major ion species including $\mathrm{H}^{+}, \mathrm{He}^{+}$and $\mathrm{O}^{+}$, with energies from $\sim 0-40 \mathrm{keV} / \mathrm{e}$ [Rème et al., 2001].

\section{Observations}

[7] Pc2 waves were recorded by the Cluster spacecraft near 13 MLT from $0800-1430$ UT on 16 February 2004, as they traveled sunward through the high latitude dayside magnetosphere and toward the magnetosheath. This is shown in Figure 1, where the geomagnetic field lines are represented using the Tsyganenko [1996] model under actual solar wind conditions for this interval. The solar wind density was $<0.5 \mathrm{~cm}^{-3}$, solar wind dynamic pressure $<0.2 \mathrm{nT}$ and the interplanetary magnetic field (IMF) south-north component $\mathrm{Bz} \sim 0$. The magnetosphere was magnetically quiet (Dst $>-50 \mathrm{nT}, \mathrm{Kp}<2$ ) and inflated greatly with the subsolar point at $\mathrm{r}=15.8 \mathrm{R}_{\mathrm{E}}$. Kp sum over this day was 11.7 , and 27.7 and 28.7 on the preceding two days. During the wave event, the Cluster satellite constellation was always located in the northern hemisphere, although the distance from Cluster to the magnetic equatorial plane decreased from $\sim 7.2 \mathrm{R}_{\mathrm{E}}$ at 0800 UT to $\sim 4.6 \mathrm{R}_{\mathrm{E}}$ at $1400 \mathrm{UT}$. The Cluster spacecraft location in the magnetosphere was also identified and confirmed using the CIS hot ion data (not shown here).

[8] Figure 2, first to third panels, shows dynamic spectra from the Cluster 1 FGM instrument for the radial, azimuthal and compressional components respectively. Waves in the Pc2 range are clearly evident, particularly in the radial and azimuthal components and with frequency gradually decreasing from around $190 \mathrm{mHz}$ at 0800 UT to $95 \mathrm{mHz}$ at 1400 UT as Cluster moved outward toward the magnetopause. The waves were recorded on closed field lines and ceased just as Cluster approached the magnetopause. Spectra from all four satellites were very similar. The observed wave frequency is always above the local helium cyclotron frequency indicated in Figure 2 by the black curves. The white curves in Figure 2 indicate the $\mathrm{He}^{+}$cyclotron frequency mapped back to the equator. This passes through the wave emission band and supports the deduction that the waves were generated off the equator. The continuous signals at $250 \mathrm{mHz}$ are due to satellite spin period interference. We also note that the Pc2 waves present an obvious burst-like pattern which is consistent with similar modulations in the ion flux distribution shown in Figure 2 (fifth panel) where the pitch angles over 1140-1230 UT at the time of large wave amplitude range over $50^{\circ}-130^{\circ}$. The second panel from the bottom shows the particle energy, and we see that proton flux significantly increased around $3 \mathrm{keV}$ and above $7 \mathrm{keV}$ during the wave event. This is favorable for the excitation of the EMIC waves [Cornwall, 1965; Arnoldy et al., 2005]. The total density of cold electrons determined from the WHISPER experiment (not shown here) was generally below $1 \mathrm{~cm}^{-3}$ in the outer magnetosphere. The wave vectors at Cluster showed large wave normal angles with respect to the geomagnetic field and were mostly directed radially outward (not shown here). Wave polarization at Cluster was mostly left handed. The Pc2 pulsations were also recorded on the ground near the satellite footprint at Casey, Zhongshan, and Davis stations in Antarctica, with similar spectral features to those recorded at Cluster (not shown here).

[9] The Poynting flux for these waves was calculated using the magnetic and electric field data via the formula $\mathbf{S}=1$ / $\mu_{0}(\delta \mathbf{E} \times \delta \mathbf{B})$, where $\delta \mathbf{E}$ and $\delta \mathbf{B}$ denote the time-varying electric field and magnetic field respectively. The direction of the wave Poynting flux vectors is shown for Cluster 1 in the first panel of Figure 3, where it is evident that the waves were directed mainly along $\left(\sim 0^{\circ}\right.$ or $\left.\sim 180^{\circ}\right)$ the magnetic field direction. The results are consistent for all four satellites. The second to seventh panels of Figure 3 show the illustrative interval from 1350-1400 UT in more detail. The direction of the Poynting flux vectors alternated from parallel to antiparallel to the B field with a periodicity approximating 1$3 \mathrm{~min}$.

\section{Discussion}

[10] The Poynting flux indicates energy transportation by wave trains. For the Pc2 waves here, the Poynting flux vector shows a unique characteristic, appearing to alternate between parallel and antiparallel with respect to the ambient magnetic field direction (Figure 3). This signature cannot be explained by previous ideas of EMIC wave generation, as outlined below. We believe these results reveal important new 


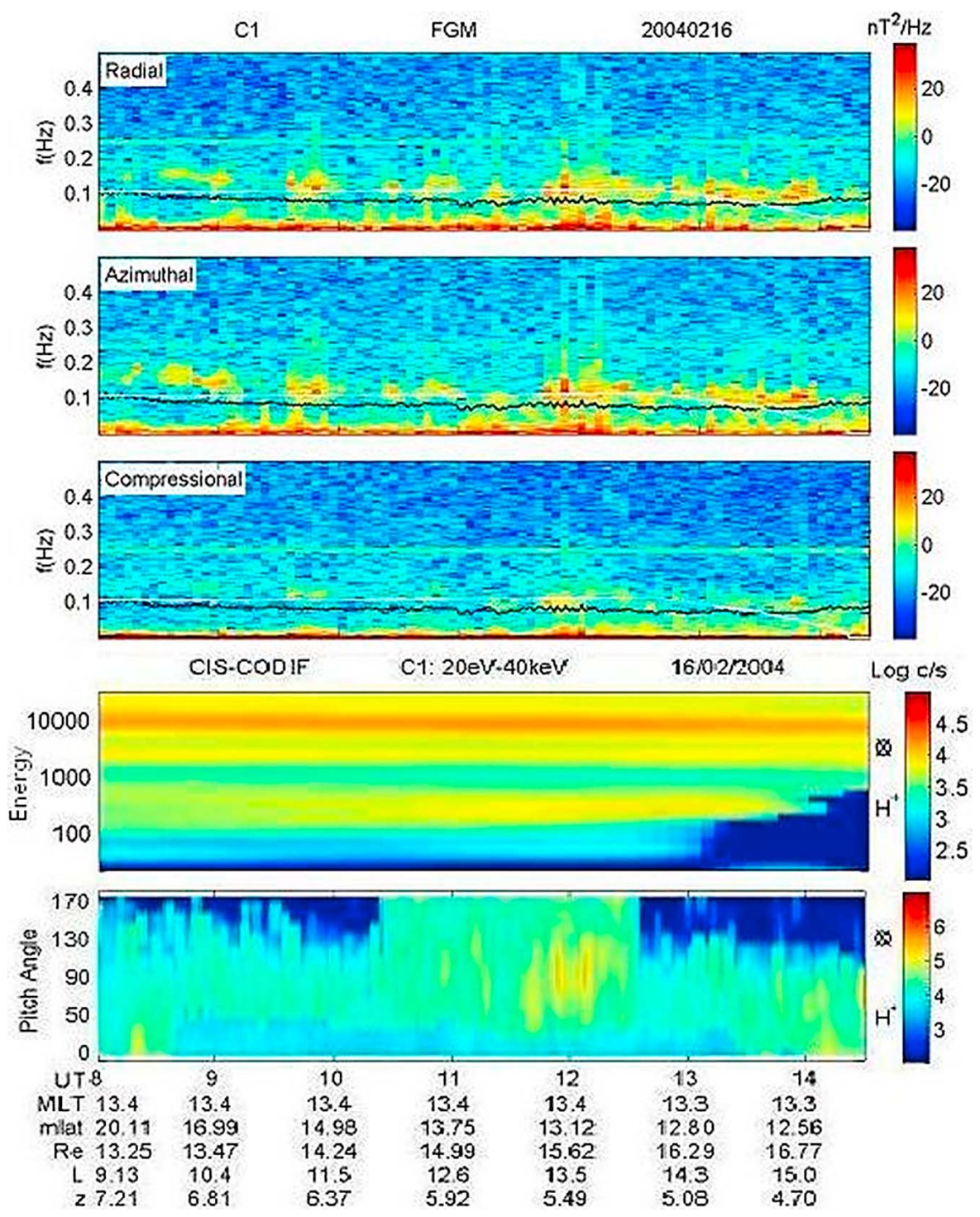

Figure 2. From top, first to third panels: dynamic spectra of Pc2 waves observed by the FGM instrument from satellite C1 over 0800-1430 UT on 16 February 2004. The observed wave frequency is always above the local helium cyclotron frequency indicated by the black line. The helium cyclotron frequency at the magnetic equator is marked by the white line. Fourth panel: energetic particle flux distribution for $\mathrm{H}^{+}$from $\mathrm{C} 1$ for the same event. The flux has been integrated over polar and azimuthal directions. Fifth panel: distribution in pitch angle of energetic particle flux for $\mathrm{H}^{+}$from $\mathrm{C} 1$ for the same event. The flux has been integrated over all energy ranges and azimuthal directions.

information on Pc2 EMIC wave generation at high latitudes in the region of minimum $\mathrm{B}$ field rather than near the magnetic equator.

[11] First we consider the possibility that the observed Pc2 waves are signatures of field line resonances (FLRs). The wave frequency decreased from 190 to $95 \mathrm{mHz}$ as Cluster moved outward from the Earth toward the magnetopause (Figure 2). Although this frequency approaches the upper end of the formal Pc3 wave frequency band, these waves do not originate from the upstream solar wind since they do not satisfy the well known formula $\mathrm{f}(\mathrm{mHz}) \approx 6 \mathrm{~B}_{\mathrm{IMF}}(\mathrm{nT})$, which generally holds for $\mathrm{Pc} 3$ waves with sources in the solar wind foreshock region. During the wave event, the interplanetary magnetic field magnitude $\left(\mathrm{B}_{\mathrm{IMF}}\right)$ was $<6.0 \mathrm{nT}$, predicting a frequency $\sim 36 \mathrm{mHz}$ for Pc3 waves, which is not seen in the observations. Furthermore, the fundamental FLR frequency at the $\mathrm{L}$ values examined here is typically around $3-5 \mathrm{mHz}$ [Liu et al., 2003]. In fact, 3-15 $\mathrm{mHz}$ Pc4-5 waves were also observed at the same time as the Pc2 event for the three components, and exhibited properties characteristic of FLRs, such as right handed polarization and significant enhanced power in all the three components [Liu et al., 2003].

[12] We next consider whether the Pc2 waves could be produced as a result of magnetospheric compression or in connection with a previous magnetic disturbance. During this interval solar wind dynamic pressure was below its normal value, only around $0.2 \mathrm{nPa}$. There was no obvious magnetic compression responsible for these waves, and magnetic conditions were quiet at the time with Dst $>-50 \mathrm{nT}$ and $\mathrm{Kp}<2$. Under similar conditions, Pc1-2 waves have been reported to occur in high $\mathrm{L}$-value regions on the ground [Engebretson et al., 2002; Popecki et al., 1993]. It has been 


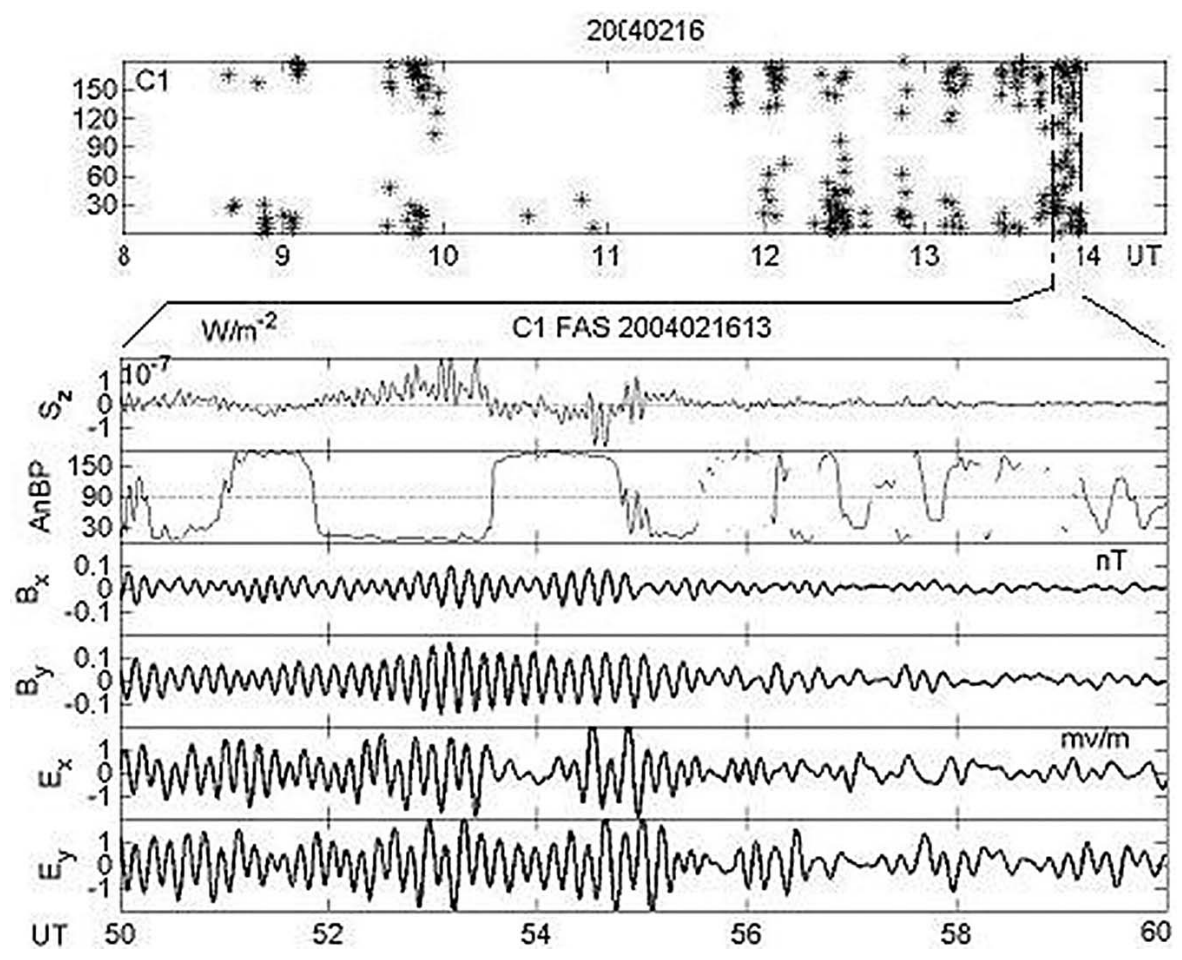

Figure 3. From top, first panel: distribution of angles between the Poynting flux vector of the Pc2 waves and the magnetic field direction during 0800-1430 UT, 16 February 2004. Second to seventh panels: the Poynting flux field aligned component $(\mathrm{Sz})$, its angles with respect to the ambient B field (AnBP), similar to that in the first panel, the $\mathrm{x}$ and y components of B field vector, and the $\mathrm{x}$ and $\mathrm{y}$ components of E field vector of the Pc2 waves during 1350-1400 UT, 16 February 2004 . All vectors are in the field aligned coordinate system. The Poynting flux vectors tend to be mainly along the magnetic field direction.

suggested that EMIC waves may be excited in the outer magnetosphere in association with enhanced density plasmasphere drainage plumes [Fraser et al., 2005]. For the Pc2 wave event here there was no obvious signature of a plasma plume seen by Cluster or by the IMAGE satellite extreme ultra-violet (EUV) images (not shown here), and there was no obvious preceding storm.

[13] It is generally believed that EMIC waves are preferably generated near and within about $15^{\circ}$ of the magnetic equator, and then propagate along field lines to higher offequator latitudes [e.g., Horne and Thorne, 1993; Loto'aniu et al., 2005]. Around the low latitude source region the wave energy transport directions are non-monotonic while beyond the source region the wave energy propagates mainly monotonically toward higher latitudes [Fraser et al., 1996]. The Pc2 wave event here was observed by Cluster around $20^{\circ}-13^{\circ}$ magnetic latitude, approximately $7.2-4.7 \mathrm{R}_{\mathrm{E}}$ north of the equatorial plane. It can be assumed that these waves were recorded at latitudes higher than any equatorial source region. However, wave energy propagated along both northward and southward field aligned directions during the entire wave event (Figure 3). Comparing the time series of the wave packets to the wave energy flux (Figure 3), it can be seen that the direction of the wave energy tended to alternate between the northward and southward directions with periodicity of 1-3 min, similar to but not exactly the same as the co-existing Pc4-5 wave frequency seen in the dynamic spectra for the Pc4-5 waves (not shown here). It is tempting to suppose the generation of these EMIC waves was modulated by Pc4-5 waves [Barfield and McPherron, 1972; Loto'aniu et al., 2009]. However, such Pc4-5 modulation can only explain the wave packet period but not the alternating energy propagation directions.

[14] The wave packet repetition period is also suggestive of equatorial wave packets bouncing between the bi-ion frequency locations or the conjugate ionospheres, i.e., the bouncing wave packet model (BWP) suggested by Jacobs and Watanabe [1964]. To test the latter notion, we make a simple estimate of the field-aligned wave packet bounce period at 1400 UT, assuming the electron number density $\mathrm{n}_{\mathrm{e}}=\sim 1 \mathrm{~cm}^{-3}$, $\rho_{\mathrm{e}}=\mathrm{n}_{\mathrm{e}} \mathrm{m}_{\mathrm{p}}$, magnetic field amplitude $\mathrm{B}=20.8 \mathrm{nT}$, and hence Alfvén velocity $\mathrm{V}_{\mathrm{A}}=\mathrm{B} /\left(\mu_{0} \rho_{\mathrm{i}}\right)^{0.5}=440 \mathrm{~km} / \mathrm{s}$. In half a packet period the wave packet can propagate $\sim 2.6$ $3.9 \times 10^{4} \mathrm{~km}$, assuming a period $1-3 \mathrm{~min}$ and a constant field aligned velocity, compared to the expected fieldaligned line path length, $\sim 2.7 \times 10^{5} \mathrm{~km}$. Therefore we rule out the possibility that the wave packets are reflected at the conjugate ionospheres.

[15] We now consider whether the wave packets are reflected from the bi-ion hybrid frequency location in the magnetosphere [Perraut et al., 1984; Guglielmi et al., 2001]. Choosing this location at $\pm 15^{\circ}$ MLat, the field line length between the two conjugate bi-ion frequency locations is around $5 \times 10^{4} \mathrm{~km}$, comparable with the distance the wave packet can travel in a single path. However, in the outer magnetosphere, the magnetic field minimum is no longer located at the equator but at higher latitude in both hemispheres since solar wind compression distorts the field line geometry. The Pc2 waves were observed at latitudes higher than $15^{\circ}$ with Poynting fluxes showing propagation in both directions. This would require the bi-ion frequency to be located at a higher latitude, say beyond $20^{\circ} \mathrm{MLat}$, in order for 
reflection at these locations to explain our observations. It is unclear if this is likely.

[16] The most likely explanation seems to be that the waves were excited in the high latitude minimum $B$ field region, which can be in the range from $\sim 15^{\circ}$ to $\sim 45^{\circ}$ MLat [Shabansky, 1971, Figure 14b]. Loto'aniu et al. [2005] had previously found an example, using Poynting flux measurements, where the EMIC source region was located within $\pm 11^{\circ}$ of the equator. Numerical calculation of the geomagnetic field and the charged particle drift shell shows the minimum magnetic field along a field line indeed occurs at high latitude in the outer magnetosphere [Shabansky, 1971] and even forms a local closed loop with constant minimum magnetic field [Antonova and Shabansky, 1968]. Such a minimum structure can trap energetic particles [Tsurutani et al., 2009; McCollough et al., 2010; Antonova et al., 2011]. Intense chorus emissions have been found to be excited in such high latitude minimum B structures [Tsurutani and Smith, 1977; Tsurutani et al., 2009]. In this paper, the Pc2 waves were observed in the outer magnetosphere with GSM $\mathrm{z} \sim 7.2-4.7 \mathrm{R}_{\mathrm{E}}$ and MLat $\sim 20-13^{\circ}$, obviously well north of the equator. The waves displayed clear signatures of both parallel and antiparallel field-aligned bouncing wave packets, and the 1-3 min bounce period requires a local reflection rather than a global bounce. These signatures encourage us to propose that the waves are excited in or near the high latitude minimum B field region. This is also supported by the Cluster CIS-CODIF data, which showed that the ion flux increased significantly in association with the Pc2 wave activity (Figure 2, fifth panel). These ion fluxes provide an obvious temperature anisotropy which should be favorable for excitation of the EMIC waves. In particular, these ions showed a clear burst pattern consistent with modulation of the Pc2 wave trains (Figure 2, fifth panel), and the pitch angles cover a range of $50^{\circ}-$ $130^{\circ}$ which includes $90^{\circ}$ pitch angles favored by particles in Shabansky orbits [McCollough et al., 2010]. Calculations using the Tsyganenko [1996] model with real solar wind data show a minimum $\mathrm{B}$ region indeed existed at high latitude in each hemisphere as marked by black asterisks in Figure 1. Therefore, we suggest these waves were generated in or near the high latitude minimum B field structure.

[17] Many previous workers have reported the observation of Pc1-2 waves with spacecraft in the outer magnetosphere [e.g., Anderson et al., 1992a, 1992b] and on the ground in the polar region, especially near the cusps [e.g., Menk et al., 1992; Zhang et. al., 2010, 2011]. It has so far been assumed that these waves are generated near the equatorial plane or in the plasma mantle on open field lines [Engebretson et al., $2005,2009]$. We show in this paper that it is more likely that EMIC wave generation occurs on distorted outer field in the minimum $B$ regions high off the equator. We believe this may be an important source region for these waves in the outer magnetosphere.

\section{Summary}

[18] In this paper we present an analysis of a Pc2 wave event which was observed by the four Cluster satellites over 0800-1400 UT on 16 February 2004 in the high latitude cusp region near local magnetic noon, from $\mathrm{L} \sim 13$ to the magnetopause at $\mathrm{L} \sim 17$. The main results are summarized as follows:
[19] 1. The waves occurred under conditions of very low solar wind density, $\rho<0.5 \mathrm{~cm}^{-3}$, and solar wind dynamic pressure, $\mathrm{P}_{\text {dyn }}<0.2 \mathrm{nPa}$, so the magnetosphere was inflated with the subsolar magnetopause around $15.8 \mathrm{R}_{\mathrm{E}}$. Magnetic conditions were quiet with no magnetic storm or compressions; Dst $>-50 \mathrm{nT}$ and $\mathrm{Kp}<2$.

[20] 2. The waves were observed in the northern hemisphere with GSM $\mathrm{z} \sim 7.2-4.7 \mathrm{R}_{\mathrm{E}}$ and MLat $\sim 20-13^{\circ}$, away from the equatorial region but on closed field lines.

[21] 3. The wave energy was dominantly transported along the field line indicating a traveling EMIC wave rather than a Field Line Resonance (FLR). Furthermore, the wave energy alternated between parallel and antiparallel to the ambient magnetic field direction, showing bouncing wave packet characteristics.

[22] 4. The ion flux associated with this wave activity increased significantly, with an obvious burst pattern signature which was highly consistent with the Pc2 wave intensity variation.

[23] 5. These results suggest that the Pc2 waves were generated locally at a high latitude minimum $B$ field region in the outer magnetosphere. We believe off-equator generation may be an important source for EMIC wave activity on distorted field lines in the outermost magnetosphere.

[24] Acknowledgments. This research was supported by Australian Research Council Discovery Project grant DP0772504 and Linkage International grant LX0882515. Y. H. L. was supported by a University of Newcastle Postgraduate Research Scholarship. Cluster satellite data has been provided by the ESA Cluster Active Archive. Now Y. H. L was supported by the Polar Research Institute of China and under the 973 Project, Ministry of Science and Technology (201005017), the Ocean Public Welfare Scientific Research Project, State Oceanic Administration, People's Republic of China (201005017) and the National Natural Science Foundation of China (40890164, 41031064).

[25] The Editor thanks Mark Engebretson and an anonymous reviewer for assisting in the evaluation of this paper.

\section{References}

Anderson, B. J., R. E. Erlandson, and L. J. Zanetti (1992a), A statistical study of Pc 1-2 magnetic pulsations in the equatorial magnetosphere: 1. Equatorial occurrence distributions, J. Geophys. Res., 97, 3075-3088, doi:10.1029/91JA02706.

Anderson, B. J., R. E. Erlandson, and L. J. Zanetti (1992b), A statistical study of Pc 1-2 magnetic pulsations in the equatorial magnetosphere: 2. Wave properties, J. Geophys. Res., 97, 3089-3101, doi:10.1029/ 91JA02697.

Antonova, E. E., I. M. Myagkova, M. V. Stepanova, M. O. Riazantseva, I. L. Ovchinnikov, B. V. Marin, and M. V. Karavaev (2011), Local particle traps in the high latitude magnetosphere and the acceleration of the relativistic electrons, J. Atmos. Sol. Terr. Phys., 73, 1465-1471, doi:10.1016/ j.jastp.2010.11.020.

Antonova, A. E., and V. P. Shabansky (1968), Structure of the geomagnetic field at great distance from the Earth, Geomagn. Aeron., Engl. Transl., 8 , 801-811.

Arnoldy, R. L., et al. (2005), Pc 1 waves and associated unstable distributions of magnetospheric protons observed during a solar wind pressure pulse, J. Geophys. Res., 110, A07229, doi:10.1029/2005JA011041.

Balogh, A., et al. (1997), The Cluster magnetic field investigation, Space Sci. Rev., 79, 65-91, doi:10.1023/A:1004970907748.

Barfield, J. N., and R. L. McPherron (1972), Investigation of interaction between Pc1-2 and Pc5 micropulsations at the synchronous orbit during magnetic storm, J. Geophys. Res., 77, 4707-4719, doi:10.1029/ JA077i025p04707.

Cornwall, J. M. (1965), Cyclotron instabilities and electromagnetic emission in the ultra low frequency and very low frequency ranges, J. Geophys. Res., 70(1), 61-69, doi:10.1029/JZ070i001p00061.

Engebretson, M. J., W. K. Peterson, J. L. Posch, M. R. Klatt, B. J. Anderson, C. T. Russell, H. J. Singer, R. L. Arnoldy, and H. Fukunishi (2002), Observations of two types of Pc 1-2 pulsations in the outer dayside magnetosphere, J. Geophys. Res., 107(A12), 1451, doi:10.1029/ 2001JA000198. 
Engebretson, M. J., T. G. Onsager, D. E. Rowland, R. E. Denton, J. L. Posch C. T. Russell, P. J. Chi, R. L. Arnoldy, B. J. Anderson, and H. Fukunishi (2005), On the source of Pc1-2 waves in the plasma mantle, J. Geophys. Res., 110, A06201, doi:10.1029/2004JA010515.

Engebretson, M. J., J. Moen, J. L. Posch, F. Lu, M. R. Lessard, H. Kim, and D. A. Lorentzen (2009), Searching for ULF signatures of the cusp: Observations from search coil magnetometers and auroral imagers in Svalbard, J. Geophys. Res., 114, A06217, doi:10.1029/2009JA014278.

Escoubet, C., M. Fehringer, and M. Goldstein (2001), Introduction: The Cluster mission, Ann. Geophys., 19, 1197-1200, doi:10.5194/angeo-191197-2001.

Fraser, B. J., and T. S. Nguyen (2001), Is the plasmapause a preferred source region of electromagnetic ion cyclotron waves in the magnetosphere?, J. Atmos. Sol. Terr. Phys., 63(11), 1225-1247, doi:10.1016/ S1364-6826(00)00225-X

Fraser, B. J., H. J. Singer, W. J. Hughes, J. R. Wygant, R. R. Anderson, and Y. D. Hu (1996), CRRES Poynting vector observations of electromagnetic ion cyclotron waves near the plasmapause, J. Geophys. Res., 101(A7), 15,331-15,343, doi:10.1029/95JA03480.

Fraser, B. J., H. J. Singer, M. L. Adrian, D. L. Gallagher, and M. F. Thomsen (2005), The relationship between plasma density structure and EMIC waves at geosynchronous orbit, in Inner Magnetosphere Interactions: New Perspectives From Imaging, Geophys. Monogr. Ser., vol. 159, edited by J. Burch, M. Schulz, and H. Spence, pp. 55-70, AGU, Washington, D. C., doi:10.1029/159GM04.

Guglielmi, A. V., J. Kangas, and A. Potapov (2001), Quasiperiodic modulation of the Pc l geomagnetic pulsations: An unsettled problem, J. Geophys. Res., 106(A11), 25,847-25,855, doi:10.1029/2001JA000136.

Gustafsson, G., et al. (2001), First results of electric field and density observations by Cluster EFW based on initial months of operation, Ann. Geophys., 19, 1219-1240, doi:10.5194/angeo-19-1219-2001.

Horne, R. B., and R. M. Thorne (1993), On the preferred source location for the convective amplification of ion cyclotron waves, J. Geophys. Res., 98(A6), 9233-9247, doi:10.1029/92JA02972.

Jacobs, J., and T. Watanabe (1964), Micropulsation whistlers, J. Atmos. Terr. Phys., 26, 825-826, doi:10.1016/0021-9169(64)90180-1.

Liu, Y. H., B. J. Fraser, R. Y. Liu, and P. Ponomarenko (2003), Conjugate phase studies of the ULF waves in the Pc5 band near the cusp, J. Geophys. Res., 108(A7), 1274, doi:10.1029/2002JA009336.

Loto'aniu, T. M., B. J. Fraser, and C. L. Waters (2005), Propagation of electromagnetic ion cyclotron wave energy in the magnetosphere, J. Geophys. Res., 110, A07214, doi:10.1029/2004JA010816.

Loto'aniu, T. M., B. J. Fraser, and C. L. Waters (2009), The modulation of electromagnetic ion cyclotron waves by Pc 5 ULF waves, Ann. Geophys., 27, 121-130, doi:10.5194/angeo-27-121-2009.
Mauk, B. H. (1982), Helium resonance and dispersion effects on geostationary Alfven/ion cyclotron waves, J. Geophys. Res., 87(A11), 9107-9119, doi:10.1029/JA087iA11p09107.

McCollough, J. P., S. R. Elkington, M. E. Usanova, I. R. Mann, D. N. Baker, and Z. C. Kale (2010), Physical mechanisms of compressional EMIC wave growth, J. Geophys. Res., 115, A10214, doi:10.1029/ 2010JA015393.

Menk, F. W., B. J. Fraser, H. J. Hansen, P. T. Newell, C.-I. Meng, and R. J. Morris (1992), Identification of the magnetospheric cusp and cleft using Pc1-2 ULF pulsations, J. Atmos. Sol. Terr. Phys., 54, 1021-1042, doi:10.1016/0021-9169(92)90069-W.

Perraut, S., R. Gendrin, A. Roux, and C. de Villedary (1984), Ion cyclotron waves: Direct comparison between ground-based measurement and observations in the source region, J. Geophys. Res., 89(A1), 195-202, doi:10.1029/JA089iA01p00195.

Popecki, M., R. Arnoldy, M. J. Engebretson, and L. J. Cahill Jr. (1993), High-latitude ground observations of Pc 1/2 micropulsations, J. Geophys. Res., 98(A12), 21,481-21,491, doi:10.1029/93JA02539.

Rème, H., et al. (2001), First multispacecraft ion measurements in and near the earth's magnetosphere with the identical cluster ion spectrometry (CIS) experiment, Ann. Geophys., 19, 1303-1354, doi:10.5194/angeo19-1303-2001.

Shabansky, V. P. (1971), Some processes in the magnetosphere, Space Sci. Rev., 12, 299-418, doi:10.1007/BF00165511.

Tsurutani, B. T., and E. J. Smith (1977), Two types of magnetospheric ELF chorus and their substorm dependences, J. Geophys. Res., 82, 5112-5128, doi:10.1029/JA082i032p05112.

Tsurutani, B. T., O. P. Verkhoglyadova, G. S. Lakhina, and S. Yagitani (2009), Properties of dayside outer zone chorus during HILDCAA events: Loss of energetic electrons, J. Geophys. Res., 114, A03207, doi:10.1029/2008JA013353.

Tsyganenko, N. A. (1996), Effects of the solarwind conditions on the global magnetospheric configuration as deduced from data-based field models, in Proceedings of the 3rd International Conference on Substorms Held in Versailles, 12-17 May 1996, edited by E. J. Rolfe and B. Kaldeich, Eur. Space Agency Spec. Publ., ESA SP-389, 181-185.

Zhang, J.-C., L. M. Kistler, C. G. Mouikis, M. W. Dunlop, B. Klecker, and J.-A. Sauvaud (2010), A case study of EMIC wave-associated $\mathrm{He}^{+}$energization in the outer magnetosphere: Cluster and Double Star 1 observations, J. Geophys. Res., 115, A06212, doi:10.1029/2009JA014784.

Zhang, J.-C., L. M. Kistler, C. G. Mouikis, B. Klecker, J.-A. Sauvaud, and M. W. Dunlop (2011), A statistical study of EMIC wave-associated $\mathrm{He}^{+}$ energization in the outer magnetosphere: Cluster/CODIF observations, J. Geophys. Res., 116, A11201, doi:10.1029/2011JA016690. 